\title{
Decoupling of coherent Gaussian beams with general astigmatism
}

\author{
J. Serna \\ Departamento de Óptica, Facultad de Ciencias Físicas, Universidad Complutense, 28040 Madrid, Spain \\ G. Nemeş* \\ E. L. Ginzton Laboratory, Stanford University, Stanford, California 94305-4085
}

Received July 2, 1993

\begin{abstract}
We show how to decouple a coherent Gaussian beam having general astigmatism (to transform it into a beam having only simple astigmatism, or orthogonal symmetry) by using a single rotated thin cylindrical lens. The resulting coherent orthogonal Gaussian beam may be further transformed into a stigmatic (rotationally symmetric) Gaussian beam by an orthogonal telescopic magnifier. The initial beam parameters must be known; we suggest a procedure for their measurement.
\end{abstract}

Coherent quadratic-phase Gaussian amplitude beams with general astigmatism and their propagation through free space, rotated orthogonal thin lenses, or nonorthogonal optical systems were studied some time ago., ${ }^{1,2}$ These beams have the minimum values for their quality parameters. ${ }^{3-5}$ We deal with only these beams in this Letter, and for simplicity we call them Gaussian beams. Partially coherent Gauss-Schell model beams with general astigmatism were also analyzed, 6,7 Using the terminology of Ref. 1, we shall refer to the beams as stigmatic (ST), simple astigmatic (SA) or orthogonal, and general astigmatic (GA) or nonorthogonal, based on whether they have rotational symmetry, two orthogonal planes of symmetry, or no orthogonal symmetry, respectively. Other names that were used are cylindrical or isotropic for the ST case and anisotropic or anamorphic ${ }^{8}$ for the SA or GA case. A Gaussian GA beam exhibits specific features. ${ }^{1,7}$ Its spot always has an elliptical shape; the ellipses of constant irradiance and the ellipses or hyperbolas of constant phase have different principal axes that rotate along the beam axis in free space. The usual parameters, such as waist and divergence, are difficult to define or less intuitive to interpret. Numerical examples for the behavior of GA beams were already presented ${ }^{1,7}$ and will not be reproduced here. GA beams are of practical interest. They are generated by nonplanar ring laser cavities $^{2,9}$ or nonplanar folded conventional cavities, or by passage of ST or SA beams through certain nonorthogonal or rotated orthogonal optical systems. ${ }^{1,10}$ Some applications (coupling to an optical fiber, a frequency-doubling configuration, drilling, cutting, thermal treatment, etc.) require SA or ST beams. In this Letter we show that it is always possible to transform a Gaussian GA beam into a SA or even a ST beam, and we find lossless passive optical systems that are able to perform such transformations (a thin cylindrical lens with appropriate power and orientation for $\mathrm{GA} \rightarrow \mathrm{SA}$; an orthogonal telescopic magnifier for SA $\rightarrow$ ST) More general beams are considered elsewhere. ${ }^{11}$ To our knowledge, a practical procedure for decoupling a GA beam has not yet been described, though this possibility was stated from a mathematical point of view. ${ }^{2,6}$

First we demonstrate the possibility of performing the transformations $\mathrm{GA} \rightarrow \mathrm{SA} \rightarrow \mathrm{ST}$ and find the corresponding optical systems; in this stage we assume that the beam parameters are known. Then we suggest a procedure to measure the necessary beam parameters for the transformation GA $\rightarrow$ SA.

A GA beam is described by an electric-field component $\Psi(x, y, z)$ having two different quadratic forms in the two variables $x, y$ for its amplitude and phase profile:

$$
\begin{aligned}
& \Psi(x, y, z)=\Psi_{0}(z) \exp \left\{-\left[x^{2} / w_{x}^{2}(z)\right.\right. \\
&+\left.\left.2 x y / w_{x y}{ }^{2}(z)+y^{2} / w_{y}^{2}(z)\right]\right\} \exp \{-j(k / 2) \\
&\left.\times\left[x^{2} / R_{x}(z)+2 x y / R_{x y}(z)+y^{2} / R_{y}(z)\right]\right\}
\end{aligned}
$$

where $z$ is the coordinate along the beam axis, $x, y$ are transverse coordinates in the laboratory $x, y, z$ frame, $k$ is the wave number in free space, and $j^{2}=$ -1 . The field described by Eq. (1) is characterized by six real parameters; $w_{x, y}$ represent the beam spot sizes (at $1 / e$ of the amplitude) along the $x, y$ axes; $R_{x, y}$ are the radii of curvature of the phase front along $x, y$; and the coefficients $1 / w_{x y}{ }^{2}$ and $1 / R_{x y}$ are specific to a GA beam. Instead of using a complex angle to describe the $x-y$ coupling, ${ }^{1}$ we use Eq. (1). The symmetry properties of the beam are thus directly related to the quadratic forms describing its amplitude and phase. Each quadratic form has its own principal axes. These are the axes in which the form contains no mixed $x y$ term; in these axes the quadratic form is said to be in its canonical, or diagonal form. The principal axes are rotated with respect to the $x, y$ frame by the angles $\theta_{w}(z)$ and $\theta_{R}(z)$, respectively (for simplicity the $z$ dependence is suppressed): 


$$
\begin{aligned}
& \tan \left(2 \theta_{w}\right)=2\left(1 / w_{x y}{ }^{2}\right) /\left(1 / w_{x}{ }^{2}-1 / w_{y}{ }^{2}\right), \\
& \tan \left(2 \theta_{R}\right)=2\left(1 / R_{x y}\right) /\left(1 / R_{x}-1 / R_{y}\right) .
\end{aligned}
$$

The GA beam has $\theta_{w} \neq \theta_{R}$ or $\theta_{w} \neq \theta_{R}+\pi / 2$ for every $z$ (the principal axes of the two quadratic forms are different). If $\theta_{w}=\theta_{R} \neq 0$ or $\theta_{w}=\theta_{R}+\pi / 2 \neq 0$ for all $z$, the beam is SA with its principal axes noncoincident with the laboratory frame $x, y$ (rotated $\mathrm{SA}$ ). For $\theta_{w}=\theta_{R}=0$, the beam is SA, having its axes along the laboratory $x, y$ axes (aligned SA, i.e., $1 / w_{x y}{ }^{2}=1 / R_{x y}=0$, but $w_{x} \neq w_{y}$ and $/$ or $R_{x} \neq R_{y}$ ). A ST beam has $1 / w_{x y}{ }^{2}=1 / R_{x y}=0$, and $w_{x}=w_{y}$, $R_{x}=R_{y}$ for all $z$. For a SA beam, there are one or two particular values of $z$ for which $w_{x}=w_{y}$ or one value of $z$ for which $R_{x}=R_{y}$. The quadratic form of the amplitude is positive definite (the beam spot is elliptical). This also represents the condition that the beam is confined near the $z$ axis.

Decoupling a GA beam means to change the beam in such a way that both its quadratic forms become canonical with respect to the same principal axes.

We can diagonalize the quadratic form of the amplitude simply by rotating about $z$ the laboratory frame from $x, y$ to $X, Y$ by the angle $\theta_{w}(z)$ (i.e., by looking at the same beam, using the frame along which the amplitude ellipse, and hence the irradiance ellipse, is aligned). In the new $X, Y$ axes (which from now on will be called the irradiance principal axes) the laser field is written as in Eq. (1) but with the lowercase $x, y$ replaced by the uppercase $X, Y$ and with $1 / w_{X Y}{ }^{2}=0$. The new parameters $w_{X}, w_{Y}, R_{X}, R_{Y}$, and $R_{X Y}$ are functions of the corresponding values in the $x, y, z$ frame. They may be easily computed by use of the appropriate rotation of the $x, y$ frame.

The next step is to act on the quadratic form describing the phase while leaving the quadratic form of the amplitude unchanged. The aim is to rotate the phase principal axes such as to coincide with the irradiance principal axes. This is achievable by a generalized thin lens, an optical element having the field transmittance (written in the new reference frame, the irradiance principal axes $X, Y)$ :

$$
t(X, Y)=\exp \left[j(k / 2)\left(X^{2} / f_{X}+2 X Y / f_{X Y}+Y^{2} / f_{Y}\right)\right] .
$$

(The following sign convention is used: $f>0$ means convergent lens action and $R>0$ means a divergent beam.) Such a lens placed at the desired location, e.g., the plane $z=z_{1}$, transforms the input field $\Psi$ into the output field $\Psi^{\prime}$ according to the law (the primed quantities describe the parameters just after the lens)

$$
\Psi^{\prime}\left(X, Y, z_{1}\right)=t(X, Y) \Psi\left(X, Y, z_{1}\right) .
$$

In order to reduce the quadratic form describing the phase it is necessary that [from Eqs. (1), (3), and (4)]

$$
f_{X Y}=R_{X Y}\left(z_{1}\right) \text {. }
$$

Therefore the field just after the generalized lens is

$$
\begin{array}{r}
\Psi^{\prime}\left(X, Y, z_{1}\right)=\Psi_{0}(z) \exp \left\{-\left[X^{2} / w_{X}{ }^{2}\left(z_{1}\right)+Y^{2} / w_{Y}^{2}\left(z_{1}\right)\right]\right\} \\
\times \exp \left\{-j(k / 2)\left[X^{2} / R_{X}^{\prime}\left(z_{1}\right)+Y^{2} / R_{Y}^{\prime}\left(z_{1}\right)\right]\right\}, \quad(6)
\end{array}
$$

where $1 / R_{X}^{\prime}\left(z_{1}\right)=1 / R_{X}\left(z_{1}\right)-1 / f_{X}$ and $1 / R_{Y}^{\prime}\left(z_{1}\right)=$ $1 / R_{Y}\left(z_{1}\right)-1 / f_{Y}$; i.e., it becomes the field of an aligned SA beam in the frame $X, Y, z$. In free-space propagation $\left(z>z_{1}\right)$ this SA beam remains SA with the same principal axes. ${ }^{1}$

The simplest generalized thin lens is a cylindrical lens having the convergence $1 / f$ along the $X$ axis and rotated by an angle $\beta$ about the $z$ axis. Its transmittance has the form of Eq. (3), with ${ }^{10}$

$$
\begin{aligned}
1 / f_{X} & =(1 / f) \cos ^{2} \beta, \\
1 / f_{Y} & =(1 / f) \sin ^{2} \beta, \\
1 / f_{X Y} & =[1 /(2 f)] \sin (2 \beta) .
\end{aligned}
$$

The conditions that have to be fulfilled by the lens are Eq. (5) and the requirement $0 \leqslant \sin 2 \beta \leqslant 1$, i.e.,

$$
|f| \leqslant(1 / 2)\left|R_{X Y}\left(z_{1}\right)\right| \text {. }
$$

The solution for decoupling a coherent GA beam is not unique. Different lenses can be used, and, once a lens has been chosen, it may decouple a given beam for several different $z_{1}$ transversal planes, with different principal axes and different mixed coefficients $1 / R_{X Y}\left(z_{1}\right)$, provided that Eqs. (5), (7c), and (8) are fulfilled at all these planes.

To perform the transformation SA $\rightarrow$ ST further, we assume that the new SA beam parameters are known (commercial devices for measuring coherent or partially coherent SA beams are currently available). The most general SA beam has different waist locations and different waist sizes on each transversal principal axis $X$ and $Y$. The ST beam may be viewed as a SA beam having both the waist locations and their sizes equal for $X$ and $Y$. The necessary optical system must act independently on the $X$ and $Y$ axes, transforming each different input waist plane into the same desired output waist plane and magnifying differently the input waist sizes to the same desired output waist size. Such an optical system has the structure $\left(F_{1}\right)\left(L_{1}\right)\left(F_{1+2}\right)\left(L_{2}\right)\left(F_{2}\right)$ for each transversal direction. Here $F$ and $L$ represent a free space and a focusing lens, respectively. The subscripts 1,2 , and $1+2$ symbolize the characteristic length of each subsystem: $f_{1}, f_{2}, f_{1}+f_{2}$, respectively. We call such a system an orthogonal telescopic magnifier. It uses four cylindrical lenses (see also Ref. 12). A simpler system, though applicable only for SA beams having the same location for both waists, was also presented..$^{13}$

Thus we have shown the possibility of performing the transformations GA $\rightarrow \mathrm{SA} \rightarrow \mathrm{ST}$, and we have found the appropriate lossless and passive optical systems. Notice that without free spaces it is not possible to reach this goal. A thin lens (spherical or cylindrical) acts only on the phase profile, leaving unchanged the irradiance profile of a beam (a beam with $w_{x} \neq w_{y}$ just before the lens will have the same irradiance parameters just after the lens).

Now we need to know how to measure the beam parameters [the angle $\theta_{w}$, which is needed for determination of the irradiance principal axes $X, Y$, and the coupling coefficient $1 / R_{X Y}\left(z_{1}\right)$ ] for the transformation GA $\rightarrow$ SA. 
One method is to measure the normalized secondorder moments of Wigner's function describing the beam, from which the desired quantities are easy to obtain for a Gaussian beam. ${ }^{14,15}$ The beam description in second order with the use of Wigner's function, the change of the second-order moments that is due to beam propagation through $A B C D$ optical systems, and the relation between the secondorder moments and the beam amplitude and phase parameters [Eq. (1)] were studied extensively. . $^{4,14-17}$ In second order, the beam is completely characterized by a $4 \times 4$ real symmetric positive-definite matrix having as elements the averages $\overline{s t}$. The overbar signifies the averaging procedure; $s$ and $t$ are any of the four-dimensional phase-space coordinates $(x, y, u, v)$ that define the position $(x, y)$ and the slope $(u, v)$ of a ray belonging to the beam. ${ }^{4-6,14-17}$ For a coherent quadratic-phase Gaussian beam this matrix is proportional to a symplectic matrix. ${ }^{14,15}$

First we need to measure the second-order moments of the beam irradiance, $\overline{x^{2}}, \overline{y^{2}}$, and $\overline{x y}$ (in the laboratory frame). They can be measured directly in any plane $z_{1}=$ constant with existing transversal beam profiles and appropriate computing algorithms. Then, using Eq. (2a), we can calculate the angle $\theta_{w}\left(z_{1}\right)$. The next measurements are made in the irradiance principal axes $X, Y$. To find the coupling coefficient $1 / R_{X Y}\left(z_{1}\right)$, we use the fact that the matrix of the second-order moments is proportional to a symplectic matrix and that $(\overline{X Y})_{1}=0$ in the irradiance principal axes $X, Y$ (Refs. 14 and 15):

$$
\begin{aligned}
1 / R_{X Y}\left(z_{1}\right) & =(\overline{X V})_{1} /\left(\overline{X^{2}}\right)_{1}=(\overline{Y U})_{1} /\left(\overline{Y^{2}}\right)_{1} \\
& =(\overline{X V}+\overline{Y U})_{1} /\left(\overline{X^{2}}+\overline{Y^{2}}\right)_{1} .
\end{aligned}
$$

The last equality is important since the term $(\overline{X V}+$ $\overline{Y U})$ is measurable. Using the free-space propagation law, ${ }^{4-6,14-17}$ we relate the term $(\overline{X V}+\overline{Y U})$ to the $\overline{X Y}$ moment (for any two planes $z_{m}, z_{n}$, where $z_{m}<z_{n}$ ) by the formula

$$
\begin{aligned}
(\overline{X Y})_{n}= & (\overline{X Y})_{m}+\left(z_{n}-z_{m}\right)(\overline{X V}+\overline{Y U})_{m} \\
& +\left(z_{n}-z_{m}\right)^{2}(\overline{U V})_{m},
\end{aligned}
$$

where the subscripts $m, n$ correspond to the planes $z_{m}, z_{n}$, respectively. Thus, measuring $\overline{X Y}$ as well as $z_{2}-z_{1}$ and $z_{3}-z_{1}$ at three different planes $z_{1,2,3}$, where $z_{1}<z_{2}<z_{3}$, we are able to obtain $(\overline{X Y}+\overline{Y U})_{1}$ at $z_{1}$.

The procedure for decoupling a GA coherent beam thus has the following steps:

(1) Select a transversal plane $z_{1}=$ constant, and find the principal axes of the irradiance ellipse at that plane $X, Y$ by measuring the second-order moments of the irradiance $\overline{x^{2}}, \overline{y^{2}}$ and $\overline{x y}$ at $z_{1}$ and computing $\theta_{w}\left(z_{1}\right)$, using Eq. (2a).

(2) Select two other locations $z_{2}, z_{3}$; measure the distances $z_{2}-z_{1}, z_{3}-z_{1}$ and the corresponding mixed moment $(\overline{X Y})_{2,3}$; find the term $(\overline{X V}+\overline{Y U})_{1}$, using Eq. (10) twice, for $n=2,3$ and $m=1$; and determine the mixed coefficient $1 / R_{X Y}\left(z_{1}\right)$, using Eq. (9).

(3) Select a cylindrical lens according to relations (5) and (8), find its rotation angle $\beta$ according to Eq. (7c), and place the lens in $z_{1}$ at the appropriate angle $\beta$ with respect to the $X$ axis.

In summary, we have shown that coherent quadratic-phase Gaussian GA beams can be transformed into SA beams by use of a simple cylindrical lens with appropriate focal length and placement. The solution is not unique. The resulting SA beam can be transformed into a ST beam by use of an orthogonal telescopic magnifier. The GA beam parameters that must be known and suggestions for their measurement have been presented also. For partially coherent, non-quadratic-phase, or non-Gaussian amplitude beams, this method is no longer valid. ${ }^{11}$

J. Serna acknowledges a grant from the Spanish Programa Nacional de Formacion de Personal Investigator and financial support from the Comisión Interministerial de Ciencia y Tecnología of Spain under Project ROB 90-539. He thanks P. M. Mejías and R. Martínez-Herrero for their help and valuable suggestions. G. Nemeş acknowledges his sponsorship by International Research and Exchanges Board, the National Science Foundation (grant ECS92-09081), and the U.S. Air Force Office of Scientific Research (contract F49620-92-J-0229). He thanks A. E. Siegman for the excellent working conditions in his laboratory.

*Permanent address, Department of Lasers, Institute of Atomic Physics, P.O. Box MG-6, Bucharest, Romania.

\section{References}

1. J. A. Arnaud and H. Kogelnik, Appl. Opt. 8, 1687 (1969)

2. J. A. Arnaud, Bell Syst. Tech. J. 49, 2311 (1970).

3. A. E. Siegman, Proc. Soc. Photo-Opt. Instrum. Eng. 1224, 2 (1990).

4. J. Serna, R. Martínez-Herrero, and P. M. Mejías, J. Opt. Soc. Am. A 8, 1094 (1991).

5. M. J. Bastiaans, Optik 88, 163 (1991).

6. R. Simon, E. C. G. Sudarshan, and N. Mukunda, Phys. Rev. A 31, 2419 (1985).

7. J. Serna, P. M. Mejías, and R. Martínez-Herrero, Opt. Quantum Electron. 24, S873 (1992).

8. D. DeJager and M. Noethen, Appl. Opt. 31, 2199 (1992).

9. A. C. Nillson, E. K. Gustafson, and R. L. Byer, IEEE J. Quantum Electron. 25, 767 (1989).

10. M. Nemeş and G. Nemeş, in Electronics Technology and Reliability (Didactica, Bucharest, 1979), pp. 535-545 (in Romanian).

11. G. Nemes, in Proceedings of the Workshop on Laser Beam Characterization (Optical Society of Spain, Madrid, 1993).

12. J. Serna, P. M. Mejías, and R. Martínez-Herrero, Opt. Quantum Electron. 24, S881 (1992).

13. L. Qiang, W. Shaomin, J. Alda, and E. Bernabeu, Optik 85, 67 (1990).

14. M. J. Bastiaans, J. Opt. Soc. Am. 69, 1710 (1979).

15. M. J. Bastiaans, Opt. Quantum Electron. 24, S1011 (1992).

16. M. J. Bastiaans, J. Opt. Soc. Am. A 3, 1227 (1986).

17. M. J. Bastiaans, Optik 82, 173 (1989). 